\title{
A SIMPLE PSO-BASED METHOD FOR POWER DISTRIBUTION SYSTEM COMPONENTS RELIABILITY PARAMETERS CALIBRATION
}

\author{
Shahrokh SHOJAEIAN ${ }^{\mathbf{1}}$, Sajjad Hashemi RIZI ${ }^{\mathbf{2}}$ \\ ${ }^{1,2}$ Khomeinishahr Branch, Islamic Azad University \\ P.O.Box 84175-119, Isfahan, IRAN \\ ${ }^{1}$ Shojaeian@iaukhsh.ac.ir \\ 22Power61850@gmail.com
}

\begin{abstract}
In this paper, a proposed algorithm based on Particle Swarm Optimization (PSO) is used to present a simple method for data calibration of reliability indices in electrical power distribution networks. The main feature of the proposed method is its comprehensiveness, since the whole reliability indices can be calibrated using a proper objective function. In order to evaluate the effectiveness of the suggested algorithm, calculations are made on the well-known IEEE-RBTS Bus2 test system. The results confirm the simplicity and validation of the proposed method, and verify that by applying the proposed method, the computation speed for data calibration can be reduced as well.
\end{abstract}

Key words: Reliability assessment, Failure rate, Repair rate, PSO

\section{Introduction}

Due to growing competition over energy sales market, power distribution companies (PDCs) have been forced to deliver an acceptable power quality and reliability. On the other hand, optimal allocation of financial resources to different development of new projects, or Preventive Maintenance (PM) should guarantee both cost-benefit requirement and costumers' satisfaction. Specifically, over the past several decades, there has been a dramatic increase in investigation of modern techniques for evaluating and meeting customer expectations. The most important of these techniques is Reliability Centered Maintenance (RCM) [1-2]. This effective technique requires an accurate set of equipment's reliability parameters, which in turn, are not simple to find.

RCM is a useful technique to find the relationship between reliability indices, weather effects, and other restrictions in the form of Probability Distribution Functions (PDFs). In other words, the system's future behavior can be predicted by evaluating its past and current state. In fact, PDFs are a kind of pattern for system behavior in which all the effecting factors on reliability indices are kept [3-4]. It is worthwhile to note that the designers can simulate the system to select the best solutions for RCM confidently, before any budget allocation.

However, in the presence of incorrect or missing data, the aforementioned process is not only practical, but also it does not provide satisfactory results. For instance, one reason for restricted use of RCM-based methods is uncertainty about the accuracy of the results. In other words, the mismatch of the computational results with the reality, which is caused by different reasons such as human mistakes, causes unacceptable consequences. Hence many efforts have been made to assess the accuracy and to validate the PDC' databases $[5,6,7]$.

In [5], first, deviations in the equipment failure rate were deliberately made. Then using the partial derivative technique, the sensitivity of the reliability indices relative to the changes was calculated, and finally it has been attempted to adjust the historical indices to the true ones. This idea has been further developed in [7-9]. In [8] instead of system modelling based on just historical data, it is tried to reflect trace of PDFs of parameters having effect on equipment's failure rates, which is obtained from inspection data

(C) 2021 Published by University Press. This is an open access article under the CC BY-NC-ND license (http://creativecommons.org/licenses/CC-BY-4.0/) 
and reach a more accurate model for the system under study. As a result, a more accurate data calibration can be done. The concept of data calibration process considered in [9], has been further discussed in [10] using the Bootstrap technique. In [11], using a threestep process, the proposed method in [5] has been developed using the concepts of confidence interval, and more samples have been considered and also probability distribution of sampling errors have been estimated, and finally the accuracy of the calibration process has been assured.

In [12], although time varying loads, the effect of Distributed Generations, and different weather conditions have been studied, the possibility of the presence of wrong or far data has not been evaluated. In [13], based on a neural network, a computing method has been presented to sensitivity analysis and reliability of Power Distribution Network (PDN). In spite of high-efficiency of the presented method, the destructive effect of incorrect data, which endangers the result, is not mentioned.

In some studies, such as [14] which are based on Reward and Penalty Scheme (RPS), uncertainty about the calculations of reliability has been considered as a financial risk. In other words, data calibration reduces uncertainty, and PDCs can obtain confirmed results to reach their goals.

Most of the above-mentioned methods are time consuming and needs heavy calculations for large PDN. Also, their importance is almost in the academic and research point of view, instead of the practical simplicity and implantability.

In this paper, instead of using some complex statistical methods or Monte Carlo simulation (MCS), a simple method based on Particle Swarm Optimization (PSO) algorithm is proposed for data calibration in refining databases of PDCs. For this purpose, first reliability indices and PSO concepts are reviewed briefly. Then, System data is also calibrated using the presented Algorithm. The presented technique here, is very effective to obtain confirmed results without complicated computations, and consequently it provides acceptable speed for calculations which are confirmed for test systems. It must be mentioned that all indices are covered with a single, Comprehensive (general) Objective Function so the newcomers are welcome and the subsequent reliability based applications can gain a more accurate answer considering all aspects (of system reliability). The main contribution of the proposed method is that it considers the PDN as a black box and equipment reliability parameters (are taken as inputs) are calibrated to minimize error between the calculated and actual system reliability indices (are taken as outputs).

\section{Reliability criteria of PDNs}

In order to evaluate the quality of service in power distribution systems, two main categories of indices are conventionally used. The first one consists of load center indices, like equipment failure rate $(\lambda)$, repair rate (r), and unavailability (U). These indices are calculated for each load center separately. The second category involves the system reliability indices, such as System Average Interruption Frequency Index (SAIFI), System Average Interruption Duration Index (SAIDI), and Customer Average Interruption Duration Index (CAIDI) [1, 6 and 15].

In addition, indices like Loss of Load Cost (LOLC), Maximum Continuous Interruption Duration (MCID), and Expected Interruption Cost (ECOST) are also specific in some countries, such as Brazil, and are not as general as previously mentioned ones [16-18].

Since the two main categories of the abovementioned indices are all average values, using traditional algorithms of reliability such as the Markov chain, along with the minimal cut-set technique can be evaluated. But for accurate evaluation of indices such as ECOST, MCID, LOLC even average values are time consuming to be computed using analytical method [15]. The most accurate method for evaluating the probability functions of all reliability indices is Monte Carlo simulation algorithm [11].

\section{Particle Swarm Optimization}

Conventionally, in a Network Control Center (NCC), the system reliability indices can and must be evaluated using actual data of the network outages. Then, real and accurate values of the system reliability indices (such as SAIFI, SAIDI, etc.) are known [19]. These reliability indices can also be calculated as a functions of the reliability parameters of the network equipment (i.e. $\lambda$ and r), theoretically [2]. There is a difference between the values of these two sets of indices. Although the former is exactly correct and actual, and the later has some estimation error, it has to be noted that usually the later has more importance in the studies, because it can give a sense about the future behavior of the system, but the former only can show what happened in the past. Approximated reliability parameters of the network equipment are determined by statistical methods and their accuracy is definitely concerned to the data gathered in the PDC databases. Always a few uncertainty and negligence are expectable in the data gathering and entry in the naturally chaotic power distribution system.

The PSO algorithm can be a good solution for calibrating inaccurate data in the power distribution network's outage database. The main idea of this paper is to calibrate the estimated reliability parameters of the network equipment, by calculating the system reliability indices, and minimizing their error compare to the actual indices evaluated in the NCC.

In the next subsections, first of all, a brief review is given for the basic concepts of PSO, then, selected system reliability indices are introduced. After that, proposed flowchart of simulation is explained and finally a case study is presented to show the effectiveness of the proposed method. 


\section{Data Calibration}

\subsection{Basic concept of PSO}

In PSO, using an iterative process, the location and velocity of the particles are updated using (1) and (2) shown below, to achieve the optimal value of the objective function (Fig. 1).

$\mathrm{X}_{i j}(\mathrm{t}+1)=\mathrm{X}_{i j}(\mathrm{t})+\mathrm{V}_{i j}(\mathrm{t}+1)$

$\mathrm{V}_{i j}(\mathrm{t}+1)=\mathrm{wV}_{i j}(\mathrm{t})+\mathrm{r}_{1} \mathrm{c}_{1}\left(P_{i j}(t)-X_{i j}(t)\right)+r_{2} \mathrm{c}_{2}\left(\mathrm{G}_{j}(\mathrm{t})-\mathrm{X}_{i j}(\mathrm{t})\right)$

Here, $w$ is the inertia coefficient, $c_{1}$ and $c_{2}$ are accelerating coefficients which are in the range of $[0$, 1], $r_{1}$ and $r_{2}$ are random coefficients in the range of [0, 1]. $\mathrm{PSO}$ is initialized with a group of random particles, and then it searches for better ones by updating the position of this particles. In each iteration, two best value are calculated. The first one is the best position, which has been achieved for each particle so far (called $P_{\text {best }}$ ), and another one is the best position which has been achieved between all of the particles so far (called $\left.\mathrm{G}_{\text {best }}\right)[20]$.

The inertial coefficient determines the effect of the particle velocity in the previous step on the current velocity. The inertia term controls the convergence behavior of PSO. Although, the inertia weight can be considered as a constant value, experimental results indicate that it is better to initially set the inertia weight to larger value and gradually reduce it to get refined solutions.

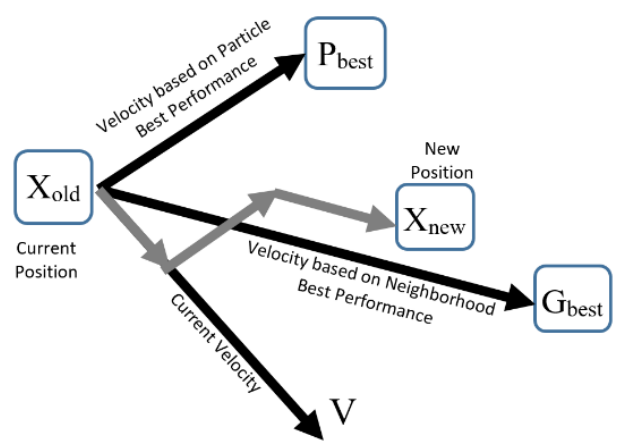

Fig. 1: General movement process of the particle in PSO

By this way, the historical values of the network equipment's reliability parameters are calibrated to their optimal value, so that the objective function (absolute difference of actual and calculated value of the system reliability indices), to be minimized.

\subsection{Indices weights and their calculation method}

To consider almost all of conventional system reliability indices of the PDN in the reliability parameters calibration, five of them have been selected in this paper (SAIFI, SAIDI, CAIDI, AENS ${ }^{1}$, and $\mathrm{ASAI}^{2}$ ). Regarding the necessity of evaluating five reliability indices in one objective function and wide range of variations, the normalizing coefficients $W_{1}$ to
$\mathrm{W}_{5}$ are considered. Therefore, different indices can be combined in a single objective function.

$$
\begin{aligned}
& W_{1}=\frac{1}{S A I F I_{a c t}} \times \alpha_{S A I F I} \\
& W_{2}=\frac{1}{S A I D I_{a c t}} \times \alpha_{S A I D I} \\
& W_{3}=\frac{1}{C A I D I_{a c t}} \times \alpha_{C A I D I} \\
& W_{4}=\frac{1}{A E N S_{a c t}} \times \alpha_{A E N S} \\
& W_{5}=\frac{1}{A S A I_{a c t}} \times \alpha_{A S A I}
\end{aligned}
$$

Here, $\alpha_{\text {SAIFI }}$ to $\alpha_{\text {ASAI }}$ are the weights for increasing effect of the corresponding indices in the objective function. In this paper, all of them take similar and equal to 1. In Equations (3)-(7), the actual reliability indices considered known, as they are available in NCC.

\subsection{Decomposition}

Decomposing the PDN into smaller sub-networks is usually done using Normally Open (N.O.) switches between adjacent feeders. From the reliability point of view, disadvantage of ignoring switching actions is limited to ignore some second order minimal cut-sets; only those cut-sets that depend on switching involving normally open nods. So, because of minor influences of these cuts, they are ignored here [11].

In this paper, in order to simplify the calculations, tie switches between adjacent feeders are assumed to be N.O. switches, without switching action during outage time. In order to extend the idea into large-scale networks, the presented method in [21] and [22] can be used.

\subsection{Objective Function}

Since this paper purpose is to provide a simple solution for PDN reliability assessment, the objective function should make decision about all of the important indices from the system designer's point of view, in the calibration process. Although just five indices are selected here, this function can be extended to all system reliability indices. The best values for unknown reliability parameters of the network equipments can be obtained, when the following objective function with five important system indices: SAIFI, SAIDI, CAIDI, AENS, and ASAI to be minimized (difference of calculated and actual values be decreased as much as possible):

$$
\begin{aligned}
& \text { Minimize : } X=W_{1} \mid\left(\text { SAIFI }_{a c t}-\text { SAIFI }_{c a l}\right) \mid+ \\
& W_{2}\left|\left(S A I D I_{a c t}-S A I D I_{c a l}\right)\right|+W_{3}\left|\left(C A I D I_{a c t}-C A I D I_{c a l}\right)\right|+ \\
& W_{4} \mid\left(A E N S_{a c t}-\text { AENS }_{c a l}\right)\left|+W_{5}\right|\left(\text { ASAI }_{a c t}-A S A I_{c a l}\right) \mid \\
& \lambda \& \mathrm{r} \text { upper band }=+25 \% * \text { actual value }
\end{aligned}
$$

\footnotetext{
${ }^{1}$ Average Energy Not Supplied
}

\footnotetext{
${ }^{2}$ Average Service Availability Index
} 
Obviously, this objective function contains a sum of the weighted absolute difference between the actual and calculated values of the system reliability indices. Ideally, it has to be converged to zero, but in practice, PSO algorithm tries to minimize it as small as possible, by changing the reliability parameters of the components $(\lambda, r)$ iteratively. After converging PSO, it can be deduced that the reliability parameters of the components are enough accurate. To saving time and accelerating PSO algorithm, two constraints are considered. They limit calculated values of the reliability parameters of the components in each step, to $\pm 25 \%$ of the database values of the corresponding parameters. Maximum number of iterations, population size (Swarm size), inertia coefficient, damping ratio have taken as 500, 22, 0.6721, and 0.8, respectively.

Fig. 2 shows the main flowchart of the proposed method. In the main loop, the particles speed and position are optimized towards minimizing the objective function. The particles best personal experience vs. iteration no. graph, indicates the efficiency of the proposed algorithm and the accuracy of the results.

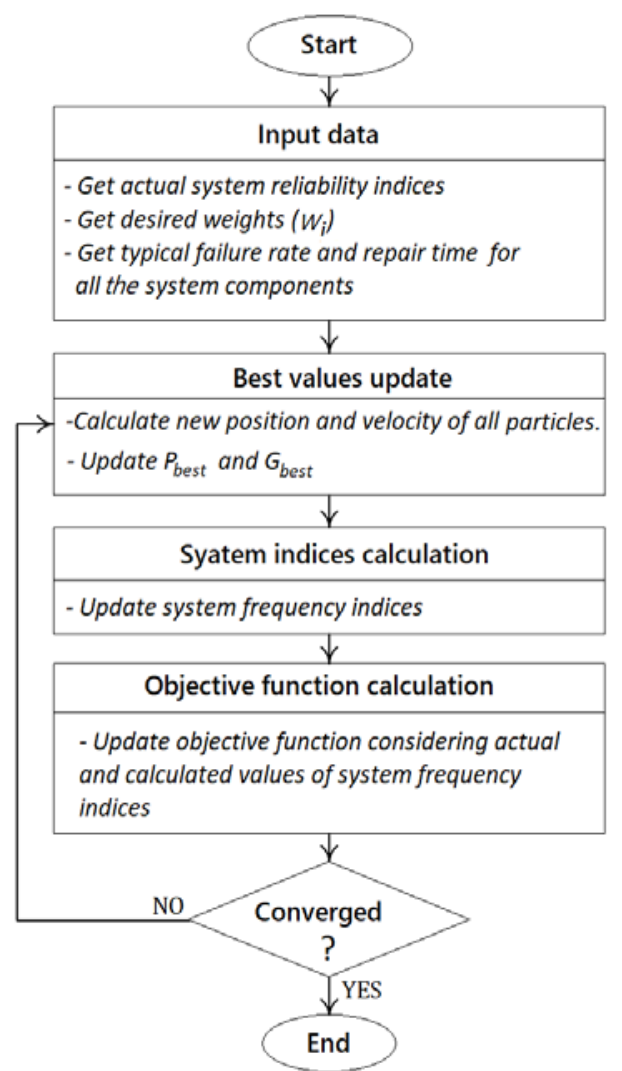

Fig. 2: Flowchart of the proposed method

\section{Simulation Results}

In order to evaluate the efficiency of the suggested algorithm, simulations are done for the Roy Billinton
Test System (RBTS) which is a $11 \mathrm{kV}$ PDN with 22 loads and 2 tie lines [23]. This test system is a wellknown network, which is used in simulation studies of many previous papers and its data is simply available. Without loss of generality, in this system only failure rate and repair time of the overhead lines are considered, because lines have higher risk of outages, compared to the other components of the system. All computations are performed in MATLAB $^{\circledR}$ using computer with an Intel core-i7 2.6 GHz processor.

\subsection{Test system}

All the information required for the IEEE-RBTS Bus2 network, including the failure and repair rates, number and type of customers, load level per load center, etc., are listed in [23]. Fig. 3 illustrates it. The proposed method has been tested on feeder R1 (Fig.1). However, as discussed earlier, in this paper switches (500 and 501) assumed to be N.O. as they are barely used to reduce the outage time using the adjacent feeder (R2). In this figure, feeders supplying areas are separated using dashed lines.

In Table 1, the required detailed data for calculations are represented. The average feeder load is $3.645 \mathrm{MW}$ and 652 different customers are fed by it. All the cutout fuse at the tee-point in lateral distributors considered fully reliable, in this case a short circuit on a lateral distributor causes its appropriate fuse to blow. This causes disconnection of its load center until the failure has to be repaired but does not affect or cause the disconnection of any other load center [2]. The busbar voltage level $11 \mathrm{KV}$ and the failure rate of all lines in single-state weather conditions is 0.065 \#/year, the repair time and the switching time are 5 and 1 hour respectively.

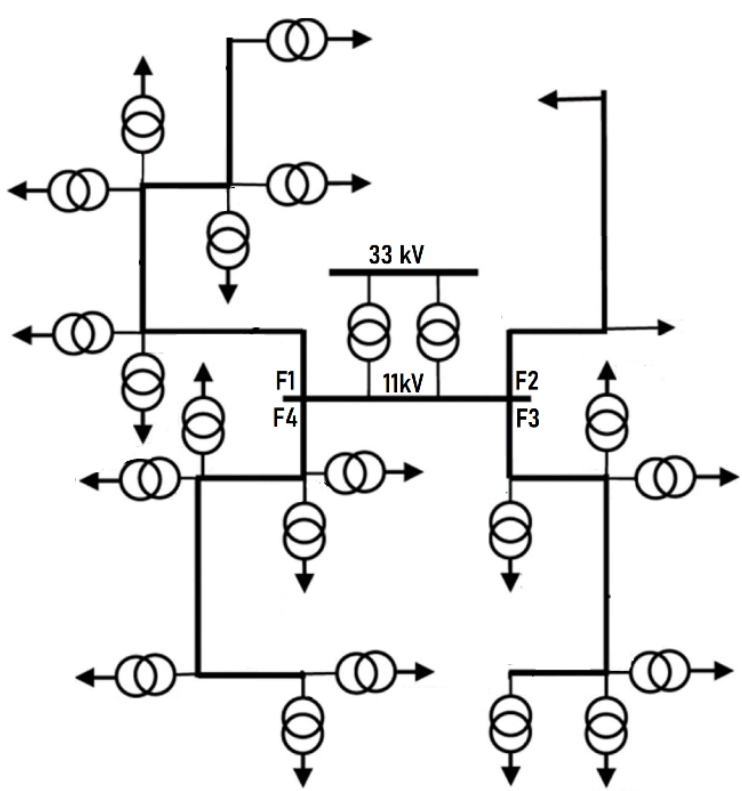

Fig. 3: The IEEE-RBTS Bus2 PDN [23]

Table 1: Load points data [23]

\begin{tabular}{ccc}
$\begin{array}{c}\text { Load } \\
\text { Point }\end{array}$ & $\begin{array}{c}\text { Average consumption } \\
\text { (MW) }\end{array}$ & $\begin{array}{c}\text { No. of } \\
\text { customers }\end{array}$ \\
\hline
\end{tabular}



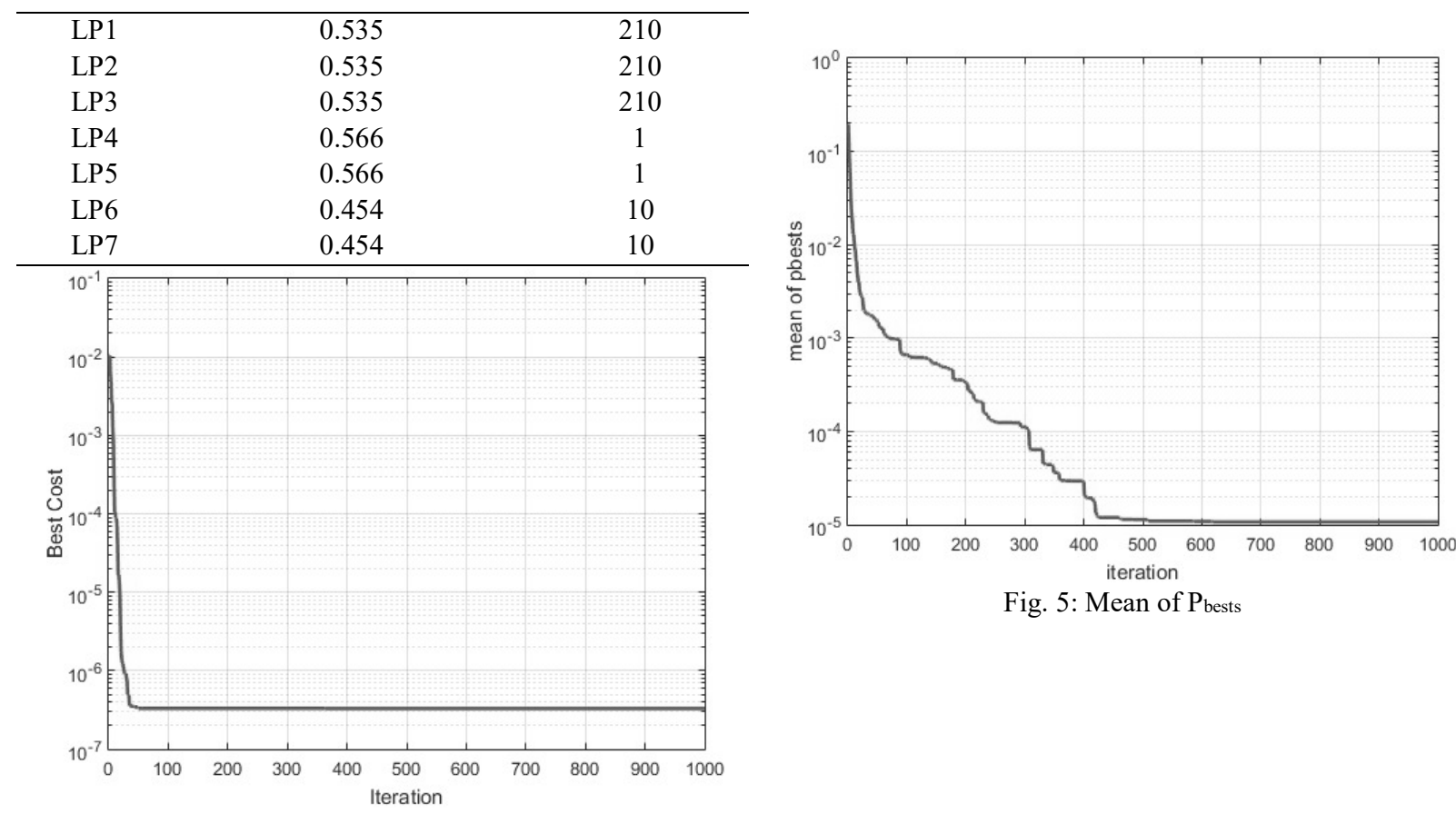

Fig. 5: Mean of $\mathrm{P}_{\text {bests }}$

Fig. 4: PSO objective function convergence diagram (No. of iterations $=1000$, final value $=3.29 * 10^{-7}$ )

Table 2: IEEE-RBTS Bus2 failure and repair data

\begin{tabular}{c|ccc|c|ccc}
\hline \multirow{2}{*}{ element } & \multicolumn{3}{|c|}{ Failure rate } & \multirow{2}{*}{ element } & \multicolumn{3}{c}{ Repair rate } \\
\cline { 2 - 3 } & database & calibrated & Adoption (\%) & & database & calibrated & Adoption (\%) \\
\hline$\lambda_{1}$ & 0.07 & 0.07 & 93 & $\mathrm{r}_{1}$ & 4 & 4.4 & 90 \\
\hline$\lambda_{2}$ & 0.07 & 0.07 & 98 & $\mathrm{r}_{2}$ & 4 & 4.4 & 92 \\
$\lambda_{3}$ & 0.07 & 0.06 & 88 & $\mathrm{r}_{3}$ & 4 & 4.8 & 84 \\
\hline$\lambda_{4}$ & 0.07 & 0.07 & 100 & $\mathrm{r}_{4}$ & 4 & 4.9 & 82 \\
$\lambda_{5}$ & 0.24 & 0.26 & 94 & $\mathrm{r}_{5}$ & 14.9 & 13.2 & 87 \\
\hline$\lambda_{6}$ & 0.24 & 0.20 & 78 & $\mathrm{r}_{6}$ & 14.9 & 13.1 & 86 \\
$\lambda_{7}$ & 0.24 & 0.22 & 91 & $\mathrm{r}_{7}$ & 14.9 & 13.1 & 88 \\
\hline$\lambda_{8}$ & 0.24 & 0.26 & 94 & $\mathrm{r}_{8}$ & 14.9 & 15.0 & 100 \\
$\lambda_{9}$ & 0.24 & 0.25 & 97 & $\mathrm{r}_{9}$ & 14.9 & 15.6 & 96 \\
\hline$\lambda_{10}$ & 0.24 & 0.24 & 98 & $\mathrm{r}_{10}$ & 14.9 & 16.5 & 91 \\
$\lambda_{11}$ & 0.24 & 0.27 & 90 & $\mathrm{r}_{11}$ & 14.9 & 17.5 & 85 \\
\hline
\end{tabular}

In order to model, test, and verify the operation of the failure and repair rate database using the proposed method, the basic reliability parameters in default database is deviated deliberately, and 11 deviated elements and uncertainties by considering $\pm 25 \%$ deviation compared to valid data of failure rate and repair time data is applied.

Table 3: IEEE-RBTS Bus2 system, R1 feeder indices

\begin{tabular}{cccc}
\hline index & $\begin{array}{c}\text { Actual } \\
\text { value }\end{array}$ & $\begin{array}{c}\text { database } \\
\text { value }\end{array}$ & $\begin{array}{c}\text { calibrated } \\
\text { value }\end{array}$ \\
\hline SAIFI (f/y) & 1.94 & 1.2 & 1.94 \\
\hline SAIDI (f/y) & 26.33 & 20.2 & 26.332 \\
\hline CAIDI (h/f) & 13.5 & 12.2 & 13.573 \\
\hline $\begin{array}{c}\text { AENS } \\
(\mathrm{kWh} / \mathrm{y})\end{array}$ & 147.2 & 140.3 & 147.2 \\
\hline ASAI & 0.9969 & 0.9969 & 0.9969 \\
\hline
\end{tabular}

\section{Results}

As shown in Figs. 4 and 5, using the proposed algorithm, the objective function obtains an acceptable convergence in less than 300 iterations, and the deviated failure rate and repair time in database are calibrated in about 65 seconds. Calibrated data of the failure and repair rates of the system are represented in Table 1. In the next step, to evaluate the accuracy of the proposed method, five system reliability indices are calculated for feeder $R_{1}$ based on the calibrated as well as uncalibrated (database) network components reliability parameters $(\lambda, r)$, and both results are compared to the actual values. Tables 2 and 3 show considered $\lambda, r$ for the network components and the resultant system indices comparison, respectively. As can be seen in these tables, system indices corresponding to the calibrated values are desirably close to the actual values. 
Due to the simplicity and generality of this method, it is possible to calibrate any system indices defined in [1]. It is worthwhile to note that the presented method can also improve the results of proposed methods in [12], the neural network [13], and other algorithms based on RPS [14], and it is suggested for the future studies.

\section{Conclusion}

Using PSO algorithm, in this article we tried to calibrate PDC's outage database for future use in reliability studies. Instead, previous works in this field, complex statistical procedures are set aside. Bear in mind keeping the data related to More Event Day, as an important part of database. On the other hand, regarding that Reliability calculations are a handy tool for system designers, the calculation speed is an important factor. Employing proposed method on a system with 11 destroyable elements and uncertainties around failure and repair rate, the objective function converged in less than 200 iterations and 65 seconds. Another advantage for using this method is its generality in a way that all known reliability indices can be analyzed in the form of a single objective function. Since PDCs use failure and repair data as a base for network improvement and PM activities. Data calibration in this field will help the system designers to get more accurate and reliable answers and be sure about the results of their plans. In brief, the main advantage of the proposed method to the previous works is that it looks into the system as a black box, so that, independent of its nature and detail, inputs (equipment reliability parameters) are calibrated to minimize error of the calculated outputs (system reliability indices) with respect to their available actual value.

\section{Acknowledgement}

The authors would like to thank the Charmahal and Bakhtiari Electric PDC, Shahrekord, for its helps in data gathering.

\section{References}

[1] Billinton, R. \& Allan, R., (1996). Reliability Evaluation of Power Systems (2 ${ }^{\text {nd }}$. Ed.) New York: Plenum Press.

[2] Brown, R., Hanson, A., Willis, H., Luedtke, F., \& Born, M. (2001) Assessing the Reliability of Distribution Systems, IEEE Computer Applications in Power, 14 (1), pp. 44-49.

[3] Asgarpoor, S., \& Mathine, M. (1995) Distribution System Reliability Evaluation with Aging Equipment, Department of Electrical Engineering and Center for Communication and Information Science, Lincoln: University of Nebraska-Lincoln.

[4] Bhargava, C., Murty, P., \& Sreevani, P. (2013) Reliability Assessment of Radial Distribution
System Incorporating Weather Effects, International Journal of Engineering Research and Development, 6 (7), pp. 45-51.

[5] Brown, R.E. \& Ochoa, J.R. (1998) Distribution System Reliability: Default Data and Model Validation, IEEE Transactions on Power System, 13(2), pp. 704-709

[6] Brown, R. (2008) Electric Power Distribution Reliability, Boca Raton: CRC Press.

[7] Da Silva, M. \& Rodrigues, A. (2013) Reliability Data Calibration Based on Load Point Interruption Indices Using Nonlinear and Quadratic Optimization, IEEE Power Tech, Grenoble.

[8] Brown, R., Frimpong, G., \& Willis, H. (2004) Failure Rate Modeling Using Equipment Inspection Data, IEEE Transactions on Power Systems, 19(2), 782-787.

[9] Guimarães, A., (2006) Distribution System Reliability: Data Calibration and Performance Based Rates, M.Sc. Dissertation, Unifei: Federal University of Itajubá

[10] Rodrigues, A. \& Da Silva, M., (2013) Confidence Intervals Estimation for Reliability Data of Power Distribution Equipments Using Bootstrap, IEEE Transactions on Power System, 28 (3), 3283-3291.

[11] Da Silva, A., Guimarães, A. \& Nascimento, L. (2014) Distribution Reliability: Data Calibration Based on Monte Carlo Simulation and Evolutionary Optimization, International Conference on Probabilistic Methods Applied to Power Systems (PMAPS), Durham.

[12] Jorge De Oliveira E Sousa, B., Humayun, M., Pihkala, A., Millar R. \& Lehtonen, M., (2017) Block-Layer Reliability Method for Distribution Systems Under Various Operating Scenarios, IEEE Transactions on Power Systems, 32 (2), pp. 978-988

[13] Tian, H., Wang, H., Wan, T., Wang, Y., Li, H., Li, J., Kang, T., \& Wang, T. (2017) A Methodology of Computing Sensitivity of Distribution System Reliability with Respect to Driving Factors Based on a BP Neural Network, Dianli Xitong Baohu Yu Kongzhi, Power System Protection and Control, 45(19), pp. 71-77

[14] Simab, M., \& Moradkhani, A. (2019) Risk-Based Switch Placement in Electric Distribution Network in Presence of Performance Based Regulation, Scientia Iranica, 21406, pp. 1-27.

[15] IEEE Std 1366-2003 (2004) IEEE Guide for Electric Power Distribution Reliability Indices

[16] Chernysheva A., Adamec V., Tulsky V., Vanin A. (2019) Calibration of the distribution network reliability model based on failure data. Case Study, International Youth Conference on Radio 
Electronics, Electrical and Power Engineering (REEPE), pp. 1-5

[17] Leite Da Silva, A., Manso, L., Mello J. \& Billinton, R. (2000) Pseudo-Chronological Simulation for Composite Reliability Analysis with Time Varying Loads, IEEE Transactions on Power System, 15(1), pp. 73-80

[18] Resolution No. 24 (2014) Aneel-Brazilian Electricity Regulatory, Available at: www.Aneel.Gov.Br

[19] Adesina L. M., Abdulkareem A., Ogunbiyi O., Ibrahim O. (2020) On-load measurement method for the reliability of distribution transformers, MethodsX, pp. 89-101

[20] Zemzami, M., Makhloufi, A., Elhami, N., Elhami, A., Itmi, M. \& Hmina, \& N. Applying (2017) A New Parallelized Version of PSO Algorithm for Electrical Power Transmission.
IOP Conference Series: Materials Science and Engineering.

[21] Leite Da Silva, A., Cassula A. \& Sacramento C., (2002) Reliability Evaluation of Distribution Systems Under Load Transfer Restrictions, 7th International Conference on Probabilistic Methods Applied to Power Systems, Naples.

[22] Leite Da Silva, A., Cassula A. Nascimento, L., Freire, J. Sacramento C. \& Guimarães, A., (2006) Chronological Monte Carlo-Based Assessment of Distribution System Reliability, 9th International Conference on Probabilistic Methods Applied to Power Systems, Stockholm.

[23] Tiwary A., Tiwary S. (2021) Evaluation of Reliability Indices of Roy Billinton Test System, Reliability: Theory \& Applications, 1 (61), pp. 54-61 